\title{
Zur Epistemologie der Natur/Kultur-Grenze und zu ihren disziplinären Folgen
}

\author{
Von Albrecht Koschorke (Konstanz)
}

\section{ABSTRACT}

Der Aufsatz behandelt ein altes Thema: die Grenzziehung zwischen Natur und Kultur. Da beide Begriffe in der Moderne zunehmend autonom gebraucht werden (Kultur ohne spezifizierendes Attribut, Natur ohne Bezug auf Gott), kann die Einheit ihrer Unterscheidung nur bewahrt werden, indem man eine Asymmetrie zwischen ihnen herstellt. Allerdings stellt sich dann die Frage, ob Natur oder Kultur als der größere und umfassende Term fungieren und ob die Grenze zwischen ihnen ihrerseits natürlich oder kulturbedingt ist. Der Aufsatz analysiert expansive und defensive Bewegungen, Übernahmen und Überschreitungen, aber auch den Austausch und die Wanderschaft von Konzepten auf diesem epistemologischen wie disziplinären Kampfgebiet.

The paper treats an old topic: the distinction between nature and culture. Since both terms have become autonomous in modern age (culture being used without a specifying attribute, nature without reference to God), the unity of their distinction can only be secured by creating an asymmetry between them. However, it is open to discussion whether Nature or Culture figures as the larger and embracing term and whether the borderline between the terms itself is snatural or sculturals. The paper analyzes expansionist and defensive moves, takeovers and transgressions, but also the exchange and traveling of concepts on this epistemological as well as disciplinary battleground.

I.

Die Opposition Natur-Kultur ist so geläufig und wird in der Regel so gedankenlos gebraucht, dass es wenig lohnend scheint, sich ein weiteres $\mathrm{Mal}$ mit ihr zu befassen. Wer das dennoch unternimmt, lädt sich drei Probleme auf. Zum einen muss er darlegen, was unter 'Natur' zu verstehen ist, oder genauer: wie dieser Begriff unter den unterschiedlichen historischen und erkenntnistheoretischen Vorzeichen bestimmt wurde. Zum anderen muss er die Frage beantworten, welchen Gehalt im Gegenzug der Begriff 'Kultur hat, wenn er als Antonym von 'Natur ' gefasst wird - und nicht etwa in Differenz zu Kulturlosigkeit oder zu anderen Kulturen. Und schließlich ist da noch der Bindestrich in der Mitte, der als unscheinbares Drittes der Unterscheidung die beiden Terme sowohl trennt als auch miteinander verkettet. Man ist also nicht nur mit den beiden Seiten der begrifflichen Opposition, sondern auch mit dem Modus des Unterscheidens selbst konfrontiert.

Was die Funktionsweise der Natur/Kultur-Entgegensetzung betrifft, 
scheint die Begriffsgeschichte von 'Kultur zunächst eine einfache Handreichung zu bieten. 'Kultur ist ja anfangs ein unselbständiger Ausdruck und bedarf einer Genitivergänzung, um sinnvoll zu werden: angefangen von der Agrikultur als dem lateinischen Wortursprung über die cultura animi bei Cicero bis hin zu mittelalterlich-christlichen Prägungen (cultura dolorum) ${ }^{1}$. Erst im 18. Jahrhundert wird die Kultur grammatikalisch und der Sache nach autonom. Fortan kommt sie ohne Spezifizierung aus; sie ist nicht mehr die Kultur, und das heißt alteuropäisch: die Kultivierung, die Pflege von etwas, und damit nicht mehr vorrangig auf etwas Zuhandenes, ihr Vorgegebenes, als natürlich oder göttlich Unverfügbares verwiesen. Stattdessen vermag sie sich nun auf sich selbst oder auf andere Gestaltungen ihrer selbst zu beziehen; sie wird zu einer in sich vollständigen Welt - auch wenn das Vorgängige der Natur, mehr oder minder eingestandenermaßen, eine Randbedingung kultureller Erscheinungen bleibt.

Wenn sich auf diese Weise rein sprachgeschichtlich ein wachsendes $\mathrm{Ge}$ wicht von 'Kultur innerhalb der Natur/Kultur-Dichotomie verzeichnen lässt, so scheint dieses Begriffspaar darum doch vergleichsweise einfach gebaut zu sein. Es handelt sich ja offenbar um zwei Teilmengen eines Ganzen, die sich zusammen zu $100 \%$ addieren. Kultur ist, was nicht Natur ist, und umgekehrt. Nimmt der Raum der vom Menschen geschaffenen Wirklichkeit auf unserem Planeten zu, so vermindert sich der Bereich der 'Natur dementsprechend. Die Interaktion beider Terme bleibt ein Nullsummenspiel: Nichts geht verloren, nur die Balance zwischen den Begriffen verschiebt sich.

Bei näherem Hinsehen geht diese Rechnung allerdings nicht so glatt auf. Es sind hier grundsätzlich zwei Niveaus zu unterscheiden, das Niveau der materiellen und das einer symbolischen Produktion. Erstere betrifft die ergologische Dimension der Kultur, die sich auf den Menschen als Werkzeug ge- . brauchendes Wesen bezieht. Agrikultur als Ursprung von Kultur überhaupt heißt ja nichts anderes als die Bearbeitung des natürlichen Bodens mit Hilfe von Werkzeugen, um den Ertrag an natürlicher Nahrung zu steigern. Entsprechend lässt sich die Technik, jedenfalls in vorindustriellen Gesellschaften, als Bearbeitung der Natur mit Werkzeugen verstehen. Dadurch wird natürlich Gegebenes vermehrt oder verfeinert, oder es werden Artefakte geschaffen, mit denen sich Menschen eine über die vorfindlichen Bedingungen der Natur hinausgehende Existenzform ermöglichen. Auch wenn man das Verhältnis zwischen Kultur und Natur - in Anlehnung an basale Nahrungsvorgänge - mit einem gewissen Recht als Stoffwechsel bezeichnen kann, ist darunter nicht eine Abnahme auf der einen und Zunahme auf der anderen Seite

1 Vgl. Wilhelm Perpeet, "Kultur, Kulturphilosophie ", in: Joachim Ritter (Hrsg.), Historisches Wörterbuch der Philosophie, Basel 1976, IV, Sp. 1309-1324, dort Sp. 1309 f. - Dirk Baecker, Wozu Kultur?, 3. Aufl., Berlin 2003, 45. 
zu verstehen. Kulturelle Artefakte hören ja nicht auf, aus natürlichen Substanzen zu bestehen, auch wenn sie weitgehend von Menschen geschaffen wurden.

Um einen 'Zuwachs an Kultur und eine korrelative 'Abnahme von Natur` auf materieller Ebene zu denken, wäre folglich ein komplexeres Modell von kulturellem Stoffwechsel einzuführen. Es hätte nicht ein einfaches Geben und Nehmen zwischen beiden Seiten dieser Dichotomie, sondern eine mehrstufige Verarbeitungskette von in der Natur Vorfindlichem darzustellen. Dabei würden so genannte Rohstoffe (das sind sie ja nicht von sich aus, sondern erst aus einer technischen Verwertungsperspektive) in verschiedenen Schritten zu Gebilden verarbeitet, die es in einer Natur ohne Menschen nicht gäbe. In dieser Hinsicht kann man tatsächlich sagen, dass das natürlich Beschaffene abnimmt und das unter kulturellen Vorzeichen Produzierte zunimmt, jedenfalls entlang der Oberfläche des Planeten Erde. Es ist ein Gemeinplatz, dass die menschliche Technik immer tiefer in die natürlichen Grundlagen eingreift. Allerdings bedeutet auch das nicht einfach einen 'Schwund der Natur, sondern ihre Anverwandlung und Umgestaltung. Hier zeigt sich schon, dass man auf die Dauer nicht mit einem binären Modell Natur-Kultur auskommen kann, sondern es zu einem (mindestens) ternären Modell erste Natur - kulturelle/technische Bearbeitung - zweite Natur erweitern muss.

Aber 'Kultur bedeutet ja nicht nur das technische Hervorbringen von Dingen, die in der Natur so nicht vorkommen. Entsprechend beschränkt sich das Verhältnis der Kultur zur Natur nicht auf Verbrauch oder Aufbereitung von Rohstoffen. Neben dieser Ebene der materiellen Anverwandlung gibt es eine zweite Ebene, nämlich die Ebene der symbolischen Produktion. Ein entscheidendes Definiens von menschlicher Kultur ist neben dem Werkzeuggebrauch der Symbolgebrauch. Zentral ist dafür die Sprachlichkeit des menşchlichen Verhältnisses zur Welt, die es erlaubt, nicht bloß mit materiellen Dingen umzugehen, sondern Bedeutungen zu prozessieren.

Bei Max Weber heißt es: "Kultur ist ein vom Standpunkt des Menschen aus mit Sinn und Bedeutung bedachter endlicher Ausschnitt aus der sinnlosen Unendlichkeit des Weltgeschehens " ${ }^{2}$. In der Kulturwissenschaft um 1900 , zu der auch Webers Soziologie zu zählen ist, war es mit den Worten Dirk Baeckers eine neuartige Idee,

daß die Kultur es mit selbstgeschaffenen Bedeutungswelten zu tun hat und daß der Mensch, wenn er sich in seiner Welt orientiert, auf nichts anderes stößt als auf diese selbstgeschaffenen Bedeutungswelten. ${ }^{3}$

${ }^{2}$ Max Weber, "Die ,Objektivität‘ sozialwissenschaftlicher und sozialpolitischer Erkenntnis“, in: ders., Gesammelte Aufsätze zur Wissenschaftslebre, Tübingen 1968, 146-214, dort: 180 .

3 Baecker (Anm. 1), 90. 
Wenn nun Kultur nicht nur eine Welt aus Artefakten ist, sondern auch eine vom Menschen »selbstgeschaffene Bedeutungswelt", eine Welt aus Symbolen, wie soll man sich dann einen 'Stoffwechsek vorstellen, der Naturdinge in kulturell erzeugte Symbole verwandelt?

Es empfiehlt sich, diese Frage - die Grundsatzfrage jeder Epistemologie vorsichtshalber nicht frontal, sondern über einen Umweg anzugehen. Mit Blick auf die Begriffsgeschichte von 'Kultur' wurde gesagt, dass sich in ihr eine Tendenz zur Autonomisierung dessen abzeichnet, was menschliche Kultur genannt werden kann; sie wird unabhängig von Genitivkonstruktionen, die sie als Bearbeitung bzw. Verfeinerung eines vorfindlichen Gutes charakterisieren, und vermag sich in wachsendem Maß auf sich selbst als eine Form der Welthervorbringung sui generis zu beziehen. Trifft dies nun auch auf den Aspekt von Kultur zu, den Dirk Baecker eine "selbstgeschaffene Bedeutungswelt« nennt? Wird auch die Symbolproduktion unabhängiger von dem, was in der Natur vorfindlich und insofern sunvordenklich ist?

Spätestens an dieser Stelle beschwört die Analogie von materieller und symbolischer Naturaneignung, so nützlich sie in anderen Zusammenhängen sein mag, eine ganze Reihe von schier unüberwindlichen Schwierigkeiten herauf. Erstens: Inwiefern sind Symbole Instrumente der Bearbeitung von vorfindlicher Natur? Wie soll man zwischen Natur und Kultur eine Linie ziehen und auf der einen Seite eine Liste der natürlich zuhandenen Dinge, auf der anderen Seite eine Liste der kulturell verfügbar gemachten Symbolisierungen anlegen? Zweitens: Was bedeutet das wachsende Autonomwerden von Kultur in diesem Zusammenhang? Emanzipiert sich die "selbstgeschaffene Bedeutungswelt" von der Vorfindlichkeit der Natur? Heißt das, dass höher entwickelte Gesellschaften eine Bedeutungswelt geschaffen haben, die von der vorfindlichen Natur weiter entfernt ist als in, sagen wir, Jäger- und Sammlerkulturen?

Man gelangt hier an einen Punkt, an dem die Analyse fundamentaler gesellschaftlicher Unterscheidungen in die Analyse kultureller Narrative übergeht, die man Große Erzählungen nennen kann. Es gibt ja eine große geschichtsphilosophische Erzählung, die von der Emanzipation des Menschen durch sein Vermögen zur Symbolisierung handelt: Anfangs wären die Menschen auch in ihrer Symbolproduktion durch den geringen Entwicklungsgrad ihrer Produktivkräfte gefesselt gewesen und hätten nur verzeichnen können, was die Natur ihnen gebot; erst später hätten sie die Freiheit gefunden, sich von dem unmittelbar Vorfindlichen auch ihrem Erkenntnisstand nach zu emanzipieren. Das heißt, am Anfang wären die Symbole gleichsam näher an den Sachen gewesen und hätten sich erst nach und nach Freiheitsspielräume von der Fesselung durch das Materielle erworben. Diese Große Erzählung wirkt plausibel, wenn man einen bestimmten Ausschnitt der Symbolproduktion wählt: beispielsweise all die Symbolsysteme, die überhaupt erst durch 
eine fortgeschrittene Technik welthaltig wurden und in der sozialen Kommunikation implementiert werden konnten. Das second life-Universum, das im Internet entsteht und sich dort ausdehnt und vervollständigt, ist unter diesem Blickwinkel sicherlich weiter entfernt von der vorfindlichen Natur als die Objektsprache von Stammeskulturen, die auf Subsistenzwirtschaft beruhen und von ihrer Umwelt in einer elementaren Weise abhängig sind.

Aber es gibt zu dieser Großen Erzählung, die von der Emanzipation der kulturellen Symbolproduktion spricht, ein ebenso plausibles und machtvolles Gegennarrativ: nämlich das Narrativ der Aufklärung und des wissenschaftlichen Fortschritts. Diesem Narrativ zufolge ist die symbolische Produktion früher Kulturen, niedergelegt in ihren magischen Praktiken, Mythen und religiösen Erzählungen, ein mehr oder weniger willkürliches Gespinst der Phantasie. Sie hat, zumindest nach modernen wissenschaftlichen Maßstäben, keinen oder nur einen sehr eingeschränkten und deformierten Objektbezug. Nach diesem grand récit (Lyotard) sind die vom Menschen geschaffenen Symbolwelter auf dem langen Weg vom Mythos zum Logos nicht etwa unabhängiger von der Natur geworden, sondern im Gegenteil näher an sie herangerückt - jedenfalls dort, wo sie sich auf externe Referenten beziehen.

Es ist also auf dem Niveau der Symbolproduktion nicht einfach, die Formel Natur + Kultur $=100 \%$ zur Anwendung zu bringen. Wenn man einen irgendwie beschaffenen Gleichlauf von technischer und symbolischer $\mathrm{Na}$ turaneignung annimmt, dann müsste man von einer wachsenden Autonomie kultureller Symbole gegenüber einer zurückweichenden Vorfindlichkeit von Natur ausgehen. Dann hätte es eine frühe Entwicklungsstufe der Menschheit gegeben, auf der die Menschen in naiver Einheit mit oder Nähe zur Natur gelebt hätten. Diesem sentimentalischen Narrativ, das erst die Moderne erfunden hat, steht ein Kompensationsmodell entgegen: In einer Menschheitsphase, in der der Grad technischer Naturbeherrschung gering ist, würden demnach die Menschen kompensatorisch in magischen Angst- und Allmachtsphantasien schwelgen und die naturgesetzlichen Abläufe in ihrem Wesen verkennen (so jedenfalls eine ältere, von Freud psychoanalytisch vertiefte Sichtweise), während umgekehrt wachsende Naturbeherrschung mit der Durchsetzung eines technisch nüchternen Realitätsprinzips einherginge. - Im einen Fall bewegt sich die Kultur von der Gegebenheit der Natur weg, im anderen Fall zu ihr hin.

II.

$\mathrm{Zu}$ verzeichnen ist hier eine sowohl theoretische als auch narrative Doppelkonditionierung, innerhalb deren sich die Natur/Kultur-Opposition, jedenfalls in ihrer modernen Ausprägung, bewegt. Um diesen Sachverhalt bes- 
ser zu verstehen, ist es notwendig, noch einen Schritt zurückzutreten und sich über die logische Form dieser Unterscheidung klarer zu werden.

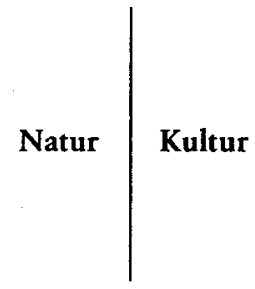

[Skizze 1]

Die erste, scheinbar rein formale Frage ist: Kann man sich Natur und Kultur durch einen geraden Trennstrich unterschieden denken? Anders gesagt: Lässt sich diese Unterscheidung als eine symmetrische Unterscheidung behandeln? Es geht hier ja um eine jener großen Unterscheidungen, die in ihrer Gesamtheit die ganze Welt umfassen. Gibt es einen epistemischen Ort, von dem aus wir sozusagen links die Natur und rechts die Kultur als in sich abgeschlossene und voneinander unabhängige Entitäten zu denken vermögen, zwischen denen eine saubere vertikale Trennung verläuft?

Das Problem besteht darin, dass eine Unterscheidung zwischen A und B, bei der A und B in der Summe 100\% ergeben, nur von einem Ort innerhalb der Unterscheidung getroffen werden kann. Die Unterscheidung ist also epistemologisch in sich selbst eingefaltet. Man könnte in diesem Fall geltend machen, die Unterscheidung sei nur von der Seite der Kultur aus zu treffen. Aber wie kommt die Menschheit im Kulturzustand dann auf die andere Seite der Dichotomie, zur Natur, wenn beide voneinander unabhängige Entitäten sind? Oder anders: Wie gelangt man zur Einheit der Unterscheidung? Eine Unterscheidung muss beides leisten, trennen und verbinden. In der Operation der Unterscheidung muss deshalb auch die Einheit der Unterscheidung ihren Ort finden, sonst zerfällt sie einfach in zwei unzusammenhängende Teile.

Ohne das an dieser Stelle eingehender begründen zu können, sei die Behauptung aufgestellt, dass ein solches Auseinanderfallen notwendig erfolgt, wenn die zwei Terme A und B sich streng symmetrisch gegenübertreten. Wenn der Trennstrich die beiden Seiten der Unterscheidung glatt teilt, dann ist zwischen ihnen keine Wechselbeziehung möglich. Solche Unterscheidungen können weder logisch noch im weiteren Sinn kulturell prozessiert werden, weil kein Weg von A nach B beziehungsweise kein Weg von A zu der Verbundenheit und Einheit von A und B führt.

Eine mögliche Antwort auf dieses Problem - oder genauer: die traditionelle Antwort auf dieses Problem - lautete ungefähr so: Die Einheit der Un- 
terscheidung kann in der Unterscheidung selbst nur dann enthalten sein, wenn sie asymmetrisch angelegt ist, das heißt aus einem großen und einem kleinen Term besteht. Dann ist der jeweils große Term dafür zuständig, den kleinen Term, dem er sich entgegenstellt, zugleich zu umgreifen und damit das umschließende Ganze der Unterscheidung zu bilden. Er wird, mit einem Ausdruck von Niklas Luhmann, als "Führgröße für die Unterscheidung selbst " verwendet ${ }^{4}$. So ist die gesamte metaphysische Tradition verfahren: Sie hat Gott und die Schöpfung, den Geist und die Natur, das Gute und das Böse als ungleiche Oppositionen behandelt, in denen der jeweils stärkere Begriff seinen schwächeren Widerpart gleichsam umklammert. Damit wurden zwei Effekte erzielt: erstens eine normative Stabilisierung zugunsten des großen und folglich hegemonialen Terms innerhalb der Opposition; zweitens die Sicherung der Einheit der Unterscheidung, weil zwei gleich große Mächte in einen unschlichtbaren manichäischen Dualismus geführt hätten. (Man sieht hier, dass die Unterscheidungslehre tiefe theologische Implikationen trägt.)

Unter postmetaphysischen Bedingungen, oder anders ausgedrückt: in modernen, dezentrierten Ordnungen, werden sich die Unterscheidungslogiken nicht mehr auf solche normativ prästabilierten Ungleichgewichte stützen können. Damit stellt sich das Problem der Einheit der Unterscheidung mit neuer Dringlichkeit, weil diese Einheit nun nicht mehr als identitär vorausgesetzt werden kann. Aber wenn die Synthesis der sozialen Welt nicht mehr durch begriffliche Hierarchien gesichert wird, in der sich die Gegensätze stufenförmig einklammern und schließlich in einem letzten, Einheit stiftenden Prinzip gipfeln - genauer: wenn ein hegemonialer Diskurs, der eine solche Synthesis behauptet, sich nicht mehr allgemein durchsetzen kann -, wie lässt sich dann verhindern, dass die Gegensätze auseinanderfallen? Haben monistische Systeme nicht immer für sich in Anschlag gebracht, dass sich ohne das Prinzip hierarchischer Inklusion die Welt in Anomie auflösen würde?

Die Lösung kann hier nicht darin liegen, dass nun die beiden Seiten der Unterscheidung gleichsam in demokratischer Gleichberechtigung nebeneinanderstehen und sich wechselseitig vollkommen relativieren. Vielmehr muss man den Blick darauf richten, dass die Asymmetrien wechseln, dass sie im Fluss sind und nicht mehr einer einzigen, alles umschließenden hegemonialen Ordnung des metaphysisch Wahren, Guten, Rechten, Eigenen gehorchen. Moderne Ordnungssemantiken stehen in der Pflicht, beweglichere Modelle zu entwickeln, als sie uns durch die philosophische Tradition zur Verfügung gestellt werden.

Aus der epistemologischen Sackgasse, in die eine strenge Dichotomie zwi-

\footnotetext{
4 Niklas Luhmann, "Kultur als historischer Begriff «, in: ders., Gesellschaftsstruktur und Semantik. Studien zur Wissenssoziologie der modernen Gesellschaft, Bd. 4., Frankfurt a.M. 1995, 31-54, hier: 48.
} 
schen Monismus und Zerfall führen würde, kommt man nur heraus, wenn man gleichsam ethnographisch vorgeht und die beterogene Beschaffenheit jedes epistemischen Feldes zum Ausgangspunkt nimmt. Nach einer solchen Sichtweise ziehen kulturelle Unterscheidungen keine gerade Linie zwischen zwei Welthälften, sondern bestehen aus zahllosen Grenzregimes lokaler oder regionaler Reichweite, die Prozesse des Austauschs, der Verhandlung und Verständigung bis zu einem gewissen Grad notwendig mit einschließen, weil Grenzen immer auch Kontaktzonen sind. In der Sprache einer ethnographisch orientierten Kultursemiotik: Teile beziehungsweise Untergruppen einer Begriffspopulation siedeln im gegenüberliegenden Feld; es gibt epistemische En- und Exklaven; verschiedene Artikulations- und Abstraktionsniveaus bilden sich aus und führenzu jeweils unterschiedlichen epistemologischen Settings, und so können immer wieder begrenzte Allianzen zwischen antagonistischen Begriffssphären entstehen. In Wahrheit sind ja auch hierarchische Stufenbauten alles andere als homogen, sondern bestehen aus vielerlei unzusammengehörigen Bauteilen.

Das bedeutet, die schlichte Totalalternative zwischen Ordnung (= Vollinklusion) und Anomie (= Null-Inklusion), zwischen Synthesis und Zerfall aufzulösen in eine dichte Beschreibung von sich überlagernden oder gegenläufigen Kohärenzen, Querverbindungen, lokalen Grenzüberschreitungen und Symbiosen, kurz: in ein bewegliches, lose gekoppeltes Gefüge von Beziehungen dritter Art. Diese Beziehungen verklammern die beiden Terme der Unterscheidung, insofern sie sie mit Asymmetrien von jeweils begrenzter Reichweite durchsetzen.

Was heißt das nun für die Unterscheidung Natur/Kultur? Aus der These der Unmöglichkeit glatt symmetrischer Unterscheidungen ergibt sich, dass auch diese Unterscheidung nur handhabbar ist, insofern man nach Formen der Asymmetrie Ausschau hält. Aus unserer Sicht liegt es nahe, unseren Beobachterstandort auf der rechten, also der Kulturseite der Unterscheidung einzunehmen. Wir würden die Unterscheidung Natur/Kultur also von der Kulturseite her asymmetrisieren. Aber wie gewinnen wir dann die Einheit dieser Unterscheidung? Anders gefragt: Wie lässt sich sicherstellen, dass $\mathrm{Na}$ tur überhaupt intelligibel ist und nicht ein absolutes Jenseits gesellschaftlicher Symbolisierungen bildet? Noch einmal anders gewendet: Auf welcher Seite der Unterscheidung ist die Natur/Kultur-Unterscheidung selbst angesiedelt?

An dieser Stelle sei eine Passage aus einem Konstanzer Forschungsprogramm zitiert, die auf Susanne Lüdemann zurückgeht:

Trotz seiner Wurzeln in der römischen Antike ist "Kultur« ein spezifisch moderner Begriff. [...] "Kultur « ist, was immer auch anders sein könnte (Nicht-Natur), und was doch nicht ohne weiteres, per Handstreich oder Verordnung, geändert werden kann ("erzwungene Wahl«; habitualisierte und institutionalisierte Handlungsroutinen als "zweite Natur ). In den letzten Jahrzehnten hat sich der Bereich dessen, was in die- 
sem Sinne als "kulturbedingt « verhandelt wird, beständig ausgeweitet: Geschlechterrollen und Formen der Wahrheitsfindung, die soziale Strukturierung von Raum und Zeit, Formen der Konfliktaustragung und der politischen oder juridischen Entscheidungsfindung, ja die Art und Weise selbst, wie in verschiedenen Gesellschaften zwischen Natur (Gegebenem, Unverfügbarem) und Kultur (Gemachtem, Verhandelbarem) unterschieden wird. ${ }^{5}$

Es geht hier nur um den letzten Teil der Überlegung - darum, dass man eine Expansion des kulturalistischen Ansatzes verzeichnen kann und dass dieser Ansatz inzwischen einschließt, dass auch die Unterscheidung "zwischen Natur (Gegebenem, Unverfügbarem) und Kultur (Gemachtem, Verhandelbarem) « eine kulturbedingte Angelegenheit ist.

Die Kultur würde hier unterscheidungslogisch die Kultur den großen, man könnte sogar sagen: hegemonialen Term bilden, der Kontrolle über die Unterscheidung als ganze, jedenfalls über die Grenzziehung innerhalb der Unterscheidung ausübt. Das Begriffspaar Natur/Kultur, so ließe sich dieser Ansatz reformulieren, ist seinerseits ein kulturelles Konzept, dessen Genese in einer Begriffsgeschichte von 'Kultur` rekonstruiert werden kann (wie wir ja hier ansatzweise auch getan haben). Dann wäre die Natur der kleine Term, diskursiv abhängig von dem, was kulturell mit ihm angestellt wird.

[Skizze 2]*

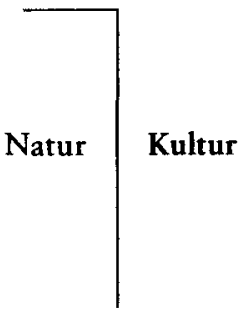

Es ließe sich aber mit gutem Grund auch eine entgegengesetzte Asymmetrisierung einführen. Man kann ja geltend machen, dass der Naturbezug kultureller Symbolisierungen umgekehrt dadurch gewährleistet wird, dass Menschen eben Naturwesen sind und dass die menschliche Kultur nur eine kleine Enklave bildet, deren Entstehung womöglich durch nichts bedingt ist als durch die Fortentwicklung natürlicher Anlagen des Menschen. Für eine solche Sichtweise wäre 'Natur` der große Term, und 'Kultur` nur eine abhängige Variable von unserer Determiniertheit durch die Natur - selbst wenn man

\footnotetext{
${ }^{5}$ Antrag auf das Exzellenzcluster Kulturelle Grundlagen von Integration, Abschnitt „Kultur als Differenzkategorie“.

Die Darstellungsweise ungleicher Unterscheidungen macht von dem durch Luhmann popularisierten Hakensymbol in Spencer Browns Laws of Form Gebrauch, verwendet ihn jedoch in einem abweichenden Sinn.
} 
einräumt, dass sich diese Abhängigkeit durch den kulturellen Fortschritt vermindert haben mag, dass es also tatsächlich eine gewisse Autonomie "selbstgeschaffener Bedeutungswelten " gegenüber den natürlichen Bedingungen gibt. Dieses Modell sähe unterscheidungslogisch so aus:

[Skizze 3]

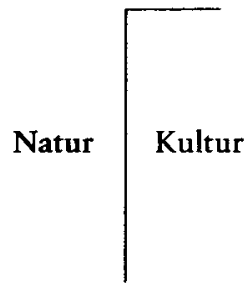

Hier sind es die natürlichen Bedingungen, unter deren Maßgabe sich Kulturen entfalten, die darüber entscheiden, was als kulturell im Sinn von sverhandelbar angesehen werden kann. - So kann man also zwei ihrerseits spiegelbildliche, d.h. symmetrische Asymmetrien anlegen, die dafür Sorge tragen, dass Natur und Kultur eine wie auch immer prekäre Einheit bilden. Der 'kulturalistische ‘ Ansatz besagt, dass Natur den Menschen nur in ihren kulturbedingten Repräsentationen zugänglich ist, dass überhaupt 'Natur' ein kulturelles Konzept ist und dass über die Frage, wo die Grenze zwischen Kultur und Natur verläuft, nach kulturspezifischen Kriterien entschieden wird. Der snaturalistische Ansatz stellt dem entgegen, dass Menschen die Reichweite ihrer kulturellen Autonomie grob überschätzen, wenn sie sich nicht der Tatsache bewusst bleiben, dass kulturelle Vorgänge gleichsam nur den verlängerten Arm natürlicher Vorgänge bilden. Das ist zwar auch eine in einem bestimmten kulturellen Zusammenhang entstandene Theorie, aber sie spricht im Namen einer Instanz, die jenseits kulturalistischer Selbstherrlichkeit liegt - eben der Natur. Beide Ansätze sind komplementär, ohne sich jedoch zu ergänzen, weil sie sich ja sozusagen wechselseitig den Boden unter den Füßen wegziehen.

Dieser Befund wird übrigens auch durch einen Blick auf die Begriffsgeschichte von Natur bestätigt. Bei aller Vielfalt der Bestimmungen, die $\curvearrowright$ Natur "von der griechischen physis und der lateinischen natura her erfährt Ritters Historisches Wörterbuch der Philosophie, das im deutschen Sprachraum zuständige Nachschlagewerk, listet unter anderem das Angeborene, das sich selbst Bewegende, "das Selbständige, Freie, Mächtige, Ursprüngliche, Gute, Vorbildliche “ auf ${ }^{6}$-, gibt es eine auffällige Parallele zur Karriere

${ }^{6}$ Friedrich Kaulbach, "Natur", in: Joachim Ritter (Hrsg.), Historisches Wörterbuch der Philosophie, Basel 1984, VI, Sp. 421-478, hier: Sp. 468. 
des Gegenbegriffs 'Kultur: nämlich das Autonomwerden beider Begriffe. Dass 'Kultur، im 18. Jh. ihre Zusätze verliert und zu einem selbständigen Substantiv wird, wurde oben erwähnt. Aber etwas ganz Ähnliches trägt sich mit der ,Natur' zu. Sie emanzipiert sich von ihrem göttlichen Schöpfer und wird zu einer eigenständigen, ihren eigenen Gesetzen folgenden und insofern geschlossenen Welt. Kant verwendet den prägnanten Ausdruck der wsich selbst überlassenen " Natur ${ }^{7}$. Im 18. Jahrhundert dankt Gott gewissermaßen ab; seither stehen sich, so scheint es, Natur und Kultur als autonome Sphären gegenüber.

Nun lässt sich leicht das Argument ins Feld führen, dass die verschiedenen Naturbegriffe, die hier nur ganz flüchtig gestreift werden konnten, schon auf Grund ihrer Varietät einen Beweis dafür liefern, dass ,Natur` keine Essenz, sondern ein kulturelles Konstrukt ist, das den jeweiligen historischen, technischen, ideellen Bedingungen ihrer Zeit unterliegt. Aber gegen dieses kulturalistische Argument würden 'Naturalisten` anführen, dass es auf jeder Stufe der Begriffsgeschichte von 'Natur` um etwas geht, das außerhalb der Reichweite kultureller Verfügbarkeit und Modellierung liegt. So würde sich also die betreffende Dichotomie auf jeder Stufe erneuern; ja man könnte paradox formulieren, dass Gesellschaften in ihrem Begriff von 'Natur symbolischen Zugang zu etwas suchen, was qua definitionem unzugänglich, der kulturellen Gestaltung vorgeschaltet und letztlich kulturell unverfügbar ist. Im Begriff 'Natur liegt also das Paradox einer symbolischen Verfügbarmachung des Unverfügbaren zutage.

III.

Weil hier nicht ontologisch, sondern kultursemiotisch argumentiert werden soll, kann die Frage, ob es dieses Unverfügbare 'gibt ‘ und wie sich wissen lässt, dass es 'da draußen ‘ ist, unentschieden bleiben. Man kann sich stattdessen darauf beschränken, zu beobachten, wie die Unterscheidung Natur/ Kultur prozessiert wird beziehungsweise wie sie ihre Widersprüche und Paradoxien austrägt, und zwar ganz offenbar, ohne dadurch entbehrlich zu werden, und welchen Grenzverlauf sie unter unterschiedlichen diskursiven Bedingungen nimmt.

Mit dieser Fragestellung öffnet sich ein unermessliches Feld. Im gegebenen Rahmen müssen einige stichwortartige Bemerkungen genügen. Zunächst einmal wäre die Fragestellung aufzuteilen. Man kann sie erstens epistemologisch akzentuieren; dann geht es um das altbekannte Problem, wie unser Wissen von den Dingen einerseits und andererseits 'die Dinge selbst،, Kantisch gesprochen: die 'Dinge an sich', zusammenhängen.

7 Ebd., Sp. 470. 
Zweitens ist die Bestimmung des Grenzverlaufs zwischen Natur und Kultur aber auch in der sozialen Praxis relevant; sie greift tief in die Organisation von Gesellschaften ein. Denn menschliche Gesellschaften sind ja selbst ein Hybrid aus Natur und Kultur - schon allein deshalb, weil sie trotz aller symbolischen Regulative auf dem Fundament sexueller Reproduktion beruhen. Auf absehbare Zeit jedenfalls hängt ihr Fortbestand noch davon ab, dass Körper sich vereinigen und fortpflanzen. Insofern stellt der menschliche Körper in allen seinen Dimensionen wohl den wichtigsten Schauplatz dar, auf dem sich der Widerstreit zwischen Natur- und Kulturreferenz zuträgt. Das betrifft den Körper als Geschlechtskörper in einer sozialen, das heißt kulturell konditionierten Ordnung; den Grenzverlauf zwischen natürlichen Gegebenheiten und kulturellen Modellierungen in Hinsicht auf sex und gender; die durch den Stand der medizinischen Technik brennend gewordene Frage, welche biologischen Indikatoren über Anfang und Ende des Menschseins entscheiden; die Sozialstruktur, insoweit sie sich auf naturbedingte Verbundenheiten zwischen Individuen beruft; schließlich das Verhältnis der Generationen zueinander und die Art und Weise, wie Gesellschaften Kontinuität durch den Transfer materieller wie spiritueller Güter sicherzustellen suchen.

So ist das Verwandtschaftssystem, ohne das bisher keine soziale Organisation ausgekommen ist, eine Kreuzung zwischen Natur und Kultur. Von den beiden Achsen jedes Verwandtschaftssystems ist die eine, die Deszendenz, mehr oder minder direkt auf natürliche Vorgänge bezogen; die andere, die Allianz, beruht dagegen auf der freien Vergesellschaftung von Menschen, nach welchen kulturellen Imperativen auch immer. Deszendenz referiert also auf die Naturseite, Allianz auf die Kulturseite von menschlichen Beziehungen, genauer: der Beziehungen zwischen menschlichen Körpern. Und gerade darum fällt auf, wie außerordentlich variationsfähig nach dem Zeugnis der ethnologischen Literatur der Verlauf dieser Grenze, je nach kultureller Ordnung, gestaltet ist.

Das Beispiel der Verwandtschaft macht evident, dass der Grenzverlauf der Natur/Kultur-Dichotomie quer durch elementare gesellschaftliche Organisationsformen geht, wobei Verwandtschaftstermini sozusagen die Grenzrelais bilden. Abstammung, Ehe, Familie sowie daraus abgeleitete Konzepte, z. B. Generation, sind hochgradig voraussetzungsreiche kulturelle Konstrukte, die jedoch - jedenfalls ihrem Selbstverständnis nach - dazu dienen, natürliche Bezüge zu ratifizieren. So wären diese Klassifikationen auf den ersten Blick getreue Abbilder vor-kultureller, natürlicher Gegebenheiten. Die Befunde der Verwandtschaftsethnologie zeigen jedoch, dass es sich nicht so einfach verhält. Zwischen biologischer Verwandtschaft und kulturbedingten Verwandtschaftstaxonomien besteht keinesfalls eine Eins-zu-eins-Beziehung. Verwandtschaftssysteme können 'natürlicher Verwandtschaften so- 
wohl annullieren als auch erzeugen. Matrilineare Systeme zum Beispiel erkennen die Verwandtschaft des Kindes mit dem Vater nicht an und erfinden sozusagen Ersatznarrative, um sie zu leugnen. Adoption hinwiederum (oder auch Patenschaft, jedenfalls im christlichen Mittelalter) ist ein rechtlicher Akt, der eine Eltern-Kind-Beziehung etabliert - häufig mit denselben Folgen wie bei biologischer Elternschaft, etwa im Hinblick auf das Inzesttabu.

Es gibt also 'natürliche، Beziehungen auf der rechten Seite der Natur/Kultur-Dichotomie, und es gibt umgekehrt kulturelle Konditionierungen dort, wo angeblich nur die Natur am Werke ist. Und die Grenze zwischen beiden ist kein trennscharfer Schnitt in einer durch große Dichotomien geordneten Welt, sondern ein drittes Feld, in dem sich entscheidet, was kulturell als natürliche Tatsache anerkannt bzw. erzeugt wird und wie umgekehrt natürliche Faktoren gegenüber kulturellen Regelungen (Leugnungen, Überschreibungen) persistieren.

Es wird hier besonders augenfällig, dass zur Beschreibung solcher Verhältnisse weder ein purer Naturalismus auf der einen Seite noch andererseits das Schlagwort der kulturellen Konstruktion ausreichend sind. Offensichtlich ist das Wissen über Verwandtschaft kulturell produziert und hängt vom jeweiligen Stand des gesellschaftlichen Wissens insgesamt ab. Vaterschaft in totemistischen Religionen ist etwas anderes als unter den Vorzeichen des römischen Rechtsdenkens, wie es der Code Napolęon erneuerte, oder im Zeitalter von DNA-Analysen und modernen Reproduktionstechnologien. Gleichwohl lässt sich kein Verwandtschaftssystem ohne Rekurs auf natürliche $\mathrm{Ge}$ gebenheiten rekonstruieren, die von den entsprechenden Klassifikationen bis zu einem gewissen Grad unabhängig sind. Wie unabhängig oder wie abhängig, lässt sich nicht ontologisch oder methodisch vorab entscheiden. Es hängt davon ab, wie der Grenzverkehr über die Natur/Kultur-Schwelle jeweils kulturell modelliert ist (oder durch natürliche Tatsachen determiniert, die auf bestimmten Entwicklungsstufen des biologischen Wissens unabweisbar werden, wie heute durch genetische Vaterschaftstests).

Ein wichtiger Relaisbegriff, der eng mit diesen verwandtschaftsterminologischen Natur-Kultur-Schaltungen verbunden ist, ist der Begriff des Erbes. Er scheint unabdingbar, um die biologische, familiensoziologische, ökonomische, moralische, ökologische und kulturelle Beziehung zwischen den Generationen zu regulieren. Auch hier zeigt sich, dass die Trennlinie innerhalb der Natur/Kultur-Dichotomie von ständigen Transfers in beiden Richtungen durchbrochen wird. Auf dem Weg einer Konzeptwanderschaft bzw. eines Konzepttausches wandern kulturell geprägte Vorstellungen von Erbschaft in die Biologie (oder ihre Vorläuferwissenschaften) ein, während umgekehrt biologische Erkenntnisse auf gesellschaftlich-kulturelle Denkweisen im Hinblick auf das Verhältnis zwischen den Zeiten und den Generationen zurückwirken. 
Die genannten Relaisbegriffe sind also nicht so sehr Grenzstationen als vielmehr Orte des Austausches zwischen den epistemischen Regimes 'Nature und 'Kultura. Es sind Orte, wo die kulturelle Semiose auf etwas von ihr Unabhängiges und Unverfügbares trifft - oder, anders betrachtet, wo das kulturelle Zeichensystem Dinge als unverfügbar externalisiert und damit dem $\mathrm{Zu}$ griff der menschlichen Willkür entzieht. Denn es ist immer ja auch eine politische Frage, was Gesellschaften als ,Natur und damit als unvordenklich ansehen und was sie als Gegenstand kultureller Aushandlungsprozesse freigeben.

Das wird noch deutlicher, wenn man das Konzept der Ethnie unter diesem Blickwinkel betrachtet. Auch Ethnien sind klassifikatorische Begriffe, die von sich behaupten, eine natürliche (oder anders, zum Beispiel herderianisch durch Sprache und Volksgeist, ontologisch begründete) Referenz zu haben. Man weiß aber, dass Verwandtschaftsgrenzen ethnische Grenzen durchkreuzen, so dass eine Vielzahl von cross-cutting ties, von Übergängen und Hybriden entsteht. Umgekehrt gibt es Verfahren der Aufnahme von Fremden in die Ethnie, Verfahren der Naturalisierung. Ethnien rangieren offenbar auf eine Ebene, die sich so weit von elementaren Vorgängen der sexuellen Reproduktion entfernt, dass man sie innerhalb der Natur/Kultur-Dichotomie vergleichsweise weit rechts eintragen muss.

Man kann daraus ersehen, wie unergiebig es ist, binäre Oppositionen ihrerseits mit binären begrifflichen Mitteln zu bearbeiten. Eine kultursemiotische Feldtheorie, wie sie hier in einer noch sehr rudimentären Form vorgestellt wurde, hätte sozusagen schon allein kartographisch mehr Möglichkeiten, Abgrenzungs- und Austauschprozesse zu beschreiben, als es das bloße Sortieren auf die linke bzw. rechte Seite der Unterscheidung bietet. Eine solche Feldtheorie könnte auch in zeitlicher Extension eine Art von sepistemischem Kampf $\triangleleft$ zwischen natürlichen Determinanten und kulturellen Klassifikationen mit seinen wechselnden Frontverläufen, Geländegewinnen und Niederlagen nachzeichnen. Aber sie könnte, und das ist wahrscheinlich noch interessanter als das Modell von Kampf und Expansion, jene Scharnierstellen und Schauplätze identifizieren, wo Konzepte ineinander transformiert werden und dabei neue Konfigurationen entstehen. Statt dem Imperativ $>\mathrm{Na}$ tur! einfach den Imperativ sulturelle Konstruktion! oder 'Kontingenz! entgegenzustellen, wären dann Prozesse der Naturalisierung und der Entnaturalisierung zu beobachten, die in ibrer Summe die Konsistenz der sozialen Wirklichkeit bilden.

Dieselbe Methode wäre auf anderen strittigen Terrains anzuwenden. Beispiele sind die nature-nurture-Debatte, der Streit über genetische Bedingtheit oder kulturelle Erworbenheit von Charaktereigenschaften, Intelligenz und Verhalten. Mit den Fortschritten der Technik rückt das Problem der Grenzziehung immer näher an den anthropologischen Kernbestand heran - 
mit Blick auf medizinische, juristische und moralische Definitionen dessen, wann menschliches Leben beginnt und endet. Immer steht hier das Natürlicher zur Disposition, und immer zeigt es sich in zwei inkommensurablen Perspektiven: einerseits als ein Substrat, auf das die kulturelle Semiosis nicht zugreifen und das sie nicht manipulieren kann oder darf; andererseits als Effekt von kulturellen Strategien der Verifikation, der Authentifizierung, der Externalisierung, der Verdinglichung, der kollektiven Beglaubigung und der kunstfertigen Herstellung von Evidenz.

IV.

Aus dem Gesagten ergibt sich eine weitaus kompliziertere Topographie der wissenschaftlichen Disziplinen, als die Rede vom great divide zwischen sciences (= Naturreferenz) und humanities (= Kulturreferenz) suggeriert. Diese Trennlinie löst sich nicht auf, aber sie vervielfacht und verästelt sich, schwächt sich in manchen Zonen $a b$ und wird in anderen hart und unüberwindlich. Aber dasselbe gilt auch für offene oder stillschweigende Austauschvorgänge oder Konzeptwanderschaften über die epistemischen Furchungen der Wissenschaftslandschaft hinweg. Das Feld wird nicht von zwei sich gegenüberstehenden Blöcken beherrscht, sondern von einem Gewimmel von Differenzen in allen Größenordnungen, die sich fortwährend neu auftun und kollabieren.

Abschließend seien einige Bemerkungen zu der Frage angefügt, inwiefern die vorgestellten Überlegungen für die Literaturwissenschaft relevant sind und was diese Disziplin zur Bearbeitung der behandelten Fragen beitragen kann:

- Erstens ist die Natur/Kultur-Dichotomie, wie gezeigt werden sollte, nur im Modus einer regulativen Fiktion zu haben und fällt damit in die Zuständigkeit einer Allgemeinen Literaturwissenschaft, die sich mit dem Zustandekommen und Funktionieren sozialer Fiktionen generell, nicht allein in poetischen Texten, beschäftigt. Näherhin sind besonders die mit dieser Dichotomie verbundenen Narrative lohnender Gegenstand einer sich literaturwissenschaftlicher Methoden bedienenden Analyse.

- Zweitens greift die jeweilige Verlaufsform der epistemischen Grenze zwischen Natur- und Kulturreferenz tief in die Methodologie der bumanities ein. Es sei nur an eine sonderbare Parallelentwicklung erinnert, die sich vielfach unbemerkt - in den vergangenen 30 Jahren zugetragen hat. Natur und 'Kultur können ja nicht für sich selbst sprechen, sondern sind auf disziplinäre Sachwalter angewiesen, die sozusagen stellvertretend einen Hegemonialkonflikt um die Vorherrschaft innerhalb der Natur/Kultur-Unterscheidung austragen. Eine solche Sachwalterschaft auf der rechten، Seite der Unterscheidung war der cultural turn der letzten Jahrzehnte. Als Kind des 
linguistic turn besteht seine dominante Theoriegester darin, auf dem Sachverhalt zu bestehen, dass uns die Wirklichkeit nicht naturaliter, sondern nur unter den Bedingungen sprachlicher und näherhin diskursiver Repräsentation gegeben ist. Das geht bis zu der bekannten und beliebten Formel von der sozialen bzw. kulturellen Produktion der Realität.

Diesem Konstruktivismus - der noch andere Ursprungsherde hat, in der Kybernetik, in der Biologie und Kognitionswissenschaft, in bestimmten philosophischen Erkenntnistheorien - steht die Dekonstruktion als ein gewissermaßen negatives Verfahren zur Seite. Sie lehrt, allen Behauptungen zu misstrauen, die sozialen Phänomenen den Charakter von Naturgegebenheit oder Wesenhaftigkeit leihen will. Ihre Theoriegeste besteht darin, die falsche, ideologisch der Festigung bestehender Machtverhältnisse verschriebene Naturalisierung, Essenzialisierung und Biologisierung sozialer Kategorien analytisch aufzubrechen. So mit Blick auf den Rassendiskurs, auf die Behauptung sozialer, ethnischer, nationaler Identität und vor allem auf die angebliche Natürlichkeit des Geschlechtergegensatzes.

Dies soll als Kurzporträt dieser theoretischen Richtung genügen. Sie scheint einen Terraingewinn der 'Kultur a auf voller Linie anzuzeigen. Erstaunlicherweise hat sich aber zur gleichen Zeit auch eine exakt gegenläufige Entwicklung abgespielt. Es sei nur ein Schlagwort genannt: die Soziobiologie. Wie der Poststrukturalismus hat sie ihre Inkubationszeit in den siebziger Jahren, aber sie tritt mit der genau gegenteiligen ,Theoriegeste hervor. Grundlage ist - in den Worten des Zoologen Edward O. Wilson - die Behauptung, dass "the humanities and social sciences shrink to specialized branches of biology; history, biography, and fiction are the research protocols of human ethology; and anthropology and sociology constitute the sociobiology of a single primate species ${ }^{8}$. Alle kulturellen Entwicklungen, so versichern die Vertreter dieser theoretischen Richtung, sind an die Leine genetischer Dispositionen genommen und sind nach den Maßgaben eines mehr oder minder elaborierten Sozialdarwinismus erklärbar.

So kam es in den siebziger Jahren zu einer symmetrischen Spaltung des epistemischen Feldes: auf der einen Seite ein szientistischer Neo-Naturalismus, der einen hegemonialen Anspruch der Biologie, insbesondere der Genetik, auf dem Feld der Geistes- und Sozialwissenschaften durchzusetzen versucht; auf der anderen Seite die Dekonstruktion und verwandte poststruktu-

${ }^{8}$ Edward O. Wilson, Sociobiology. The New Synthesis, Cambridge/Mass. 1975, 547. - Vgl. Axel Heinrich, "Gesellschaft ram langen Band der Gener (E.O. Wilson). Überlegungen zum metapolitischen Charakter der Soziobiologie", Jabrbuch für christliche Sozialwissenschaften 45 (2004), 61-82. - Dirk Richter, "Das Scheitern der Biologisierung der Soziologie. Zum Stand der Diskussion um die Soziobiologie und anderer evolutionstheoretischer Ansätze", Kölner Zeitschrift für Soziologie und Sozialpsychologie 57 (2005), 523-542. 
ralistische Strömungen, die nicht nur solche Biologismen kritisieren, sondern auch zum ,Gegenangriff übergehen: etwa in der Dekonstruktion der biologischen Geschlechterpolarität. Teils nehmen beide Richtungen polemisch aufeinander Bezug, teils ignorieren sie sich wechselseitig und bewegen sich in weitgehend getrennten Kommunikationssphären. Wie auch immer, Soziobiologie und (poststrukturalistische) Kulturwissenschaft erscheinen hier als zwei inverse Formationen, um die Natur/Kultur-Differenz einem der beiden Terme als dominantem Term zu unterstellen. Und es wird einmal mehr deutlich, dass die Differenzlinie zwischen Natur und Kultur kein harmloser Trennstrich ist, sondern die Front in einem epistemischen Kampfgebiet.

- Ein drittes Themenfeld, auf dem die Natur/Kultur-Dichotomie für die Literaturwissenschaften relevant wird, lässt sich unter die Stichworte 'Körper und Medien s subsumieren. Spätestens seit der Moderne leben die Menschen ja nicht in einer Welt natürlicher Körper, sondern einer durch Distanzmedien (allen voran: die Schrift) gleichsam entwendeten und imaginativ wiedererstatteten Körperlichkeit. Diese Transformation macht die Rede von ,Natürlichkeit bis zu einem gewissen Grad obsolet, weil das, was Menschen als ihre Natur ansehen, ihnen durch kulturelle und technische Vermittiung gegeben beziehungsweise zurückgegeben ist.

- Das letzte Terrain der Natur/Kultur-Dichotomie und ihrer vielfachen Metamorphosen, das hier angesprochen werden soll, ist schließlich die Literatur selbst. Es ist ja eine besondere Eigenschaft von Metaphern im engeren (linguistischen) und weiteren (literarischen) Sinn, beiden Verschiebungen vom Künstlichen ins Natürliche und zurück - einen Schauplatz zu bieten. Je nach ihrer Anlage können sprachliche Gebilde etwas als Natur Geltendes in den Modus der Uneigentlichkeit rücken und umgekehrt einen anfangs uneigentlich verwendeten Ausdruck mit allen Attributen der Natürlichkeit, Ursprünglichkeit, Unvordenklichkeit ausstatten. Bevorzugtes Objekt solcher sprachlichen Strategien ist der Körper in seiner sowohl individuellen als auch sozialen Dimension, der sich fast ohne Rest kulturell überschreiben oder aber als letztes Reservat einer widerständigen Authentizität adeln lässt. Dann kann der Körper sogar als Instanz des Einspruchs der Natur gegen seine zeichenhafte Darstellung in der Literatur behandelt werden. Und so trägt potentiell jeder poetische Text etwas von der paradoxen Grundspannung aus, in der kulturelle Symbolik und naturhaft Entzogenes sich zueinander befinden. 